PROCEEDINGS OF THE

AMERICAN MATHEMATICAL SOCIETY

Volume 134, Number 10, October 2006, Pages 2975-2982

S 0002-9939(06)08326-2

Article electronically published on May 4, 2006

\title{
COMMUTANTS OF CERTAIN ANALYTIC OPERATOR ALGEBRAS
}

\author{
GUOXING JI, TOMOYOSHI OHWADA, AND KICHI-SUKE SAITO
}

(Communicated by David R. Larson)

\begin{abstract}
We prove that algebraic commutants of maximal subdiagonal algebras and of analytic operator algebras determined by flows in a $\sigma$-finite von Neumann algebra are self-adjoint.
\end{abstract}

\section{INTRODUCTION}

Let $\mathcal{H}$ be a complex Hilbert space and let $\mathcal{B}(\mathcal{H})$ be the algebra of all bounded linear operators on $\mathcal{H}$. For a subset $E$ of $\mathcal{B}(\mathcal{H})$, we denote by $E^{\prime}$ the algebraic commutant, that is,

$$
E^{\prime}=\{X \in \mathcal{B}(\mathcal{H}): A X=X A, \forall A \in E\} .
$$

If $T \in \mathcal{B}(\mathcal{H})$, we call $\{T\}^{\prime}$ the algebraic commutant of $T$. The well-known theorem of Fuglede states that if $N$ is normal and $X$ commutes with $N$, so does $X^{*}$. That is, the algebraic commutant $\{N\}^{\prime}$ of $N$ is self-adjoint. Note that $\{N\}^{\prime}$ is the same as the commutant of the algebra generated by $N$ and $I$, which is non-self-adjoint in general. Thus it may be asked which subalgebras have a self-adjoint commutant. For example, if all elements in a subalgebra are normal or the algebra itself is selfadjoint, then its algebraic commutant is self-adjoint. In general, this problem is not particularly interesting. However special cases of this problem are interesting. F. Gilfeather and D.R. Larson in [6] showed that the algebraic commutant of a nest subalgebra of a von Neumann algebra is self-adjoint. We note that a nest subalgebra of a von Neumann algebra is a kind of analytic operator algebra. Thus it is interesting to consider this problem for general analytic operator algebras.

In [2, W. Arveson introduced the notion of subdiagonal algebras to give a unified theory of non-self-adjoint operator algebras, including the algebra of bounded analytic matrix-valued (or more generally, operator-valued) functions and nest subalgebras of von Neumann algebras.

Received by the editors November 2, 2004 and, in revised form, April 29, 2005.

2000 Mathematics Subject Classification. Primary 46L10, 46L55.

Key words and phrases. Von Neumann algebra, subdiagonal algebra, analytic operator algebra, algebraic commutant.

The first author was supported in part by the National Natural Science Foundation of China (No. 10571114) and the Excellent Young Teachers Program of MOE, P.R.C. The second author was supported in part by a Grant-in-Aid for Scientific Research from the Japanese Ministry of Education, Culture, Sports, Science and Technology. The third author was supported in part by a Grant-in-Aid for Scientific Research, Japan Society for the Promotion of Science. 
Let $\mathcal{M}$ be a $\sigma$-finite von Neumann algebra acting on $\mathcal{H}$. We denote by $\mathcal{M}_{*}$ the space of all $\sigma$-weakly continuous linear functionals of $\mathcal{M}$. For a von Neumann subalgebra $\mathfrak{D}$ of $\mathcal{M}$, let $\Phi$ be a faithful normal conditional expectation from $\mathcal{M}$ onto $\mathfrak{D}$. A subalgebra $\mathfrak{A}$ of $\mathcal{M}$, containing $\mathfrak{D}$, is called a subdiagonal algebra of $\mathcal{M}$ with respect to $\Phi$ if

(i) $\mathfrak{A} \cap \mathfrak{A}^{*}=\mathfrak{D}$,

(ii) $\Phi$ is multiplicative on $\mathfrak{A}$, and

(iii) $\mathfrak{A}+\mathfrak{A}^{*}$ is $\sigma$-weakly dense in $\mathcal{M}$.

The algebra $\mathfrak{D}$ is called the diagonal of $\mathfrak{A}$. Although subdiagonal algebras are not assumed to be $\sigma$-weakly closed in [2], the $\sigma$-weak closure of a subdiagonal algebra is again a subdiagonal algebra of $\mathcal{M}$ with respect to $\Phi$ (Remark 2.1.2 in [2]). Thus we assume that our subdiagonal algebras are always $\sigma$-weakly closed.

We say that $\mathfrak{A}$ is a maximal subdiagonal algebra in $\mathcal{M}$ with respect to $\Phi$ in case that $\mathfrak{A}$ is not properly contained in any other subalgebra of $\mathcal{M}$ which is subdiagonal with respect to $\Phi$. Put $\mathfrak{A}_{0}=\{X \in \mathfrak{A}: \Phi(X)=0\}$ and $\mathfrak{A}_{m}=\{X \in \mathcal{M}: \Phi(A X B)=$ $\left.\Phi(B X A)=0, \forall A \in \mathfrak{A}, B \in \mathfrak{A}_{0}\right\}$. By Theorem 2.2.1 in 2], we recall that $\mathfrak{A}_{m}$ is a maximal subdiagonal algebra of $\mathcal{M}$ with respect to $\Phi$ containing $\mathfrak{A}$. If there is a faithful normal finite trace $\tau$ on $\mathcal{M}$ such that $\tau \circ \Phi=\tau$, we say that $\mathfrak{A}$ is finite subdiagonal.

On the other hand, let $\alpha=\left\{\alpha_{t}\right\}_{t \in \mathbb{R}}$ be a flow of $\mathbb{R}$ on $\mathcal{M}$, i.e. $\left\{\alpha_{t}\right\}_{t \in \mathbb{R}}$ is a oneparameter group of *-automorphisms of $\mathcal{M}$ such that, for each $X \in \mathcal{M}, t \rightarrow \alpha_{t}(X)$ is $\sigma$-weakly continuous. Write $H^{\infty}(\alpha)=\left\{X \in \mathcal{M}: s p_{\alpha}(X) \subseteq[0, \infty)\right\}$, where $s p_{\alpha}(\cdot)$ is an Arveson spectrum (Section 3). Then $H^{\infty}(\alpha)$ is a $\sigma$-weakly closed subalgebra of $\mathcal{M}$ satisfying that $H^{\infty}(\alpha)+\left(H^{\infty}(\alpha)\right)^{*}$ is $\sigma$-weakly dense in $\mathcal{M}$. The structure of $H^{\infty}(\alpha)$ was studied by several authors (cf. [3, 13, 14, 16]). It is known that if there is a faithful normal conditional expectation from $\mathcal{M}$ onto $H^{\infty}(\alpha) \cap\left(H^{\infty}(\alpha)\right)^{*}$, then $H^{\infty}(\alpha)$ is a maximal subdiagonal algebra of $\mathcal{M}$. Moreover, if $\mathcal{A}$ is a nest subalgebra of $\mathcal{M}$, then there is an inner flow $\alpha=\left\{\alpha_{t}\right\}_{t \in \mathbb{R}}$, that is, $\alpha$ is implemented by a continuous unitary group $\left\{U_{t}: t \in \mathbb{R}\right\} \subset \mathcal{M}$, such that $\mathcal{A}=H^{\infty}(\alpha)$ (cf. Theorem 4.2.3 in 14]).

In this note we prove that algebraic commutants of maximal subdiagonal algebras and of analytic operator algebras are self-adjoint.

\section{The COMmutant of a MAXimal SUBDiAgonal AlgEBRA}

We consider the algebraic commutant of a maximal subdiagonal algebra $\mathfrak{A}$ with respect to $\Phi$. The following result was proved in [9]. For completeness, we give the proof here also.

Lemma 1. Let $\mathfrak{A}$ be a finite subdiagonal algebra with respect to $\Phi$ of $\mathcal{M}$. Then $\mathfrak{A}^{\prime}=\mathcal{M}^{\prime}$.

Proof. It is trivial that $\mathfrak{A}^{\prime} \supseteq \mathcal{M}^{\prime}$. Now let $X \in \mathfrak{A}^{\prime}$ and $T \in \mathcal{M}$. Then for any $\epsilon>0$, we have that $T^{*} T+\epsilon I$ is a positive invertible operator in $\mathcal{M}$. Note that $\mathfrak{A}$ is maximal subdiagonal (cf. [5]). By Theorem 4.2.1 in [2], there is an invertible operator $A$ in $\mathfrak{A}$ so that $T^{*} T+\epsilon I=A^{*} A$. Then

$$
X^{*}\left(T^{*} T+\epsilon I\right) X=X^{*} A^{*} A X=A^{*} X^{*} X A \leq\|X\|^{2} A^{*} A=\|X\|^{2}\left(T^{*} T+\epsilon I\right) .
$$

It follows that $X^{*} T^{*} T X \leq\|X\|^{2} T^{*} T$ by letting $\epsilon \rightarrow 0$. In particular, $X^{*} E X \leq$ $\|X\|^{2} E$ for every positive projection $E$ in $\mathcal{M}$. It follows that $(I-E) X^{*} E X(I-E) \leq$ 
0, which implies that $E X(I-E)=0$. Thus $E X=X E$ for every projection $E \in \mathcal{M}$, which implies that $X \in \mathcal{M}^{\prime}$. The proof is complete.

We next recall Haagerup's reduction theory [7. Since $\mathcal{M}$ is $\sigma$-finite, there exists a faithful normal state $\varphi$ of $\mathcal{M}$ such that $\varphi \circ \Phi=\varphi$. Let $\sigma^{\varphi}=\left\{\sigma_{t}^{\varphi}\right\}_{t \in \mathbb{R}}$ be the modular automorphism group of $\mathcal{M}$ associated with $\varphi$. We know that $\mathfrak{A}$ is $\left\{\sigma_{t}^{\varphi}\right\}_{t \in \mathbb{R}}$ invariant from Theorem 2.4 in [10. Let $G$ be the discrete subgroup $\bigcup_{n>1} 2^{-n} \mathbb{Z}$ of $\mathbb{R}$. We consider the crossed product $\mathcal{M} \rtimes_{\sigma^{\varphi}} G$ with respect to $\sigma^{\varphi}$. Then we have that $\mathcal{M} \rtimes_{\sigma^{\varphi}} G$ is a von Neumann algebra on $\ell^{2}(G, \mathcal{H})$ generated by the operators $\pi(X), X \in \mathcal{M}$, and $\lambda(s), s \in G$, defined by the equations

$$
(\pi(X) \xi)(t)=\sigma_{-t}^{\varphi}(X) \xi(t), \quad \xi \in \ell^{2}(G, \mathcal{H}), t \in G,
$$

and

$$
(\lambda(s) \xi)(t)=\xi(t-s), \quad \xi \in \ell^{2}(G, \mathcal{H}), t \in G .
$$

Note that $\pi$ is a normal faithful representation of $\mathcal{M}$ on $\ell^{2}(G, \mathcal{H})$. Let $\hat{\varphi}$ be the dual weight of $\varphi$ on $\mathcal{M} \rtimes_{\sigma^{\varphi}} G$. Then $\hat{\varphi}$ is again a faithful normal state on $\mathcal{M} \rtimes_{\sigma^{\varphi}} G$. Haagerup's reduction theorem asserts that there is an increasing sequence $\left\{\mathcal{R}_{n}\right\}_{n \geq 1}$ of von Neumann subalgebras of $\mathcal{M} \rtimes_{\sigma^{\varphi}} G$ with the following properties:

(i) each $\mathcal{R}_{n}$ is finite;

(ii) $\bigcup_{n \geq 1} \mathcal{R}_{n}$ is $\sigma$-weakly dense in $\mathcal{M} \rtimes_{\sigma^{\varphi}} G$;

(iii) for each $n \geq 1$ there is a faithful normal conditional expectation $\mathcal{E}_{n}$ from $\mathcal{M} \rtimes_{\sigma^{\varphi}} G$ onto $\mathcal{R}_{n}$ such that $\hat{\varphi} \circ \mathcal{E}_{n}=\hat{\varphi}, \quad \mathcal{E}_{n} \circ \mathcal{E}_{n+1}=\mathcal{E}_{n}, \quad n \geq 1$, and $\lim _{n \rightarrow \infty}\left\|\psi \circ \mathcal{E}_{n}-\psi\right\|=0$ for all $\psi \in\left(\mathcal{M} \rtimes_{\sigma \varphi} G\right)_{*}$.

We refer the readers to [7] and [17] for more details.

We now can extend $\Phi$ to a normal faithful conditional expectation $\hat{\Phi}$ from $\mathcal{M} \rtimes_{\sigma^{\varphi}} G$ onto $\mathfrak{D} \rtimes_{\sigma^{\varphi}} G$, which is naturally identified as a von Neumann subalgebra of $\mathcal{M} \rtimes_{\sigma^{\varphi}} G$.

Let $\hat{\mathfrak{A}}$ be the $\sigma$-weakly closed subalgebra generated by $\{\pi(X): X \in \mathfrak{A}\}$ and $\{\lambda(s): s \in G\}$. Since $\mathfrak{A}$ is $\left.\sigma_{t}^{\varphi}\right|_{t \in \mathbb{R}}$ invariant by Theorem 2.4 in $[10, \hat{\mathfrak{A}}$ is the $\sigma$-weak closure of the set of all linear combinations of $\lambda(s) \pi(X), s \in G, X \in \mathfrak{A}$. The following lemma was proved in [17].

Lemma 2. $\hat{\mathfrak{A}}$ is a maximal subdiagonal algebra with respect to $\hat{\Phi}$.

Let $\mathfrak{A}_{n}=\mathcal{R}_{n} \cap \hat{\mathfrak{A}}=\mathcal{E}_{n}(\hat{\mathfrak{A}})$. We have the following lemma (Lemma 2 in [17]).

Lemma 3. $\mathfrak{A}_{n}$ is a finite subdiagonal algebra in $\mathcal{R}_{n}$ with respect to $\left.\hat{\Phi}\right|_{\mathcal{R}_{n}}$ and $\bigcup_{n \geq 1} \mathfrak{A}_{n}$ is $\sigma$-weakly dense in $\hat{\mathfrak{A}}$.

We now have the main theorem in this section.

Theorem 1. Let $\mathcal{M}$ be a $\sigma$-finite von Neumann algebra and let $\mathfrak{A}$ be a maximal subdiagonal algebra with respect to $\Phi$ of $\mathcal{M}$. Then the commutant $\mathfrak{A}^{\prime}$ of $\mathfrak{A}$ is selfadjoint, that is, $\mathfrak{A}^{\prime}=\mathcal{M}^{\prime}$.

Proof. We first claim that $\hat{\mathfrak{A}}^{\prime}=\left(\mathcal{M} \rtimes_{\sigma^{\varphi}} G\right)^{\prime}$. In fact, let $X \in \hat{\mathfrak{A}}^{\prime}$. Then $X \in \mathfrak{A}_{n}^{\prime}$ for all $n \in \mathbb{N}$. By Lemmas 1 and 3 , we have $X^{*} \in \mathfrak{A}_{n}^{\prime}$. Note that for every $Y \in \hat{\mathfrak{A}}$, we have $\mathcal{E}_{n}(Y) \in \mathfrak{A}_{n}$ for all $n \in \mathbb{N}$ and $Y=\lim _{n} \mathcal{E}_{n}(Y) \sigma$-weakly from Haagerup's theory. Then it follows that $X^{*} Y=Y X^{*}$, which implies that $X^{*} \in \hat{\mathfrak{A}}^{\prime}$. 
Now let $X \in \mathfrak{A}^{\prime}$. We define an operator $\hat{X}$ on $\ell^{2}(G, \mathcal{H})$ by

$$
(\hat{X} \xi)(s)=X \xi(s), s \in G, \xi \in \ell^{2}(G, \mathcal{H}) .
$$

Then we have that $\hat{X} \in \hat{\mathfrak{A}}^{\prime}$. In fact, for $\xi \in \ell^{2}(G, \mathcal{H}), t, s \in \mathbb{R}$ and $Y \in \mathfrak{A}$,

$$
\begin{aligned}
(\hat{X} \pi(Y) \xi)(t) & =X(\pi(Y) \xi)(t)=X \sigma_{-t}^{\varphi}(Y) \xi(t) \\
& =\sigma_{-t}^{\varphi}(Y) X \xi(t)=\sigma_{-t}^{\varphi}(Y)((\hat{X} \xi)(t)) \\
& =(\pi(Y) \hat{X} \xi)(t)
\end{aligned}
$$

and

$$
\begin{aligned}
\left(\hat{X} \lambda_{s} \xi\right)(t) & =X\left(\left(\lambda_{s} \xi\right)(t)\right)=X \xi(t-s) \\
& =(\hat{X} \xi)(t-s)=\left(\lambda_{s}(\hat{X} \xi)\right)(t) \\
& =\left(\lambda_{s} \hat{X} \xi\right)(t) .
\end{aligned}
$$

It follows that $(\hat{X})^{*} \in \hat{\mathfrak{A}}^{\prime}$, which implies that $(\hat{X})^{*} \pi(Y)=\pi(Y)(\hat{X})^{*}$ for all $Y \in \mathfrak{A}$. Note that $(\hat{X})^{*}=\left(X^{*}\right)^{*}$. Then $\left(X^{*}\right)^{r} \pi(Y)=\pi(Y)\left(X^{*}\right)^{\wedge}$ for all $Y \in \mathfrak{A}$. In particular, $X^{*} Y=Y X^{*}$. Thus we have $X^{*} \in \mathfrak{A}^{\prime}$. Note that $\mathfrak{A}+\mathfrak{A}^{*}$ is $\sigma$-weakly dense in $\mathcal{M}$. It then follows that $X \in \mathcal{M}^{\prime}$. Hence $\mathfrak{A}^{\prime}=\mathcal{M}^{\prime}$. The proof is complete.

\section{The COMmutant of An Analytic Operator Algebra}

In this section we consider the algebraic commutant of an analytic operator algebra determined by a flow on $\mathcal{M}$. We need Arveson's theory of spectral subspaces and, so we recall the definitions here. Let $\alpha=\left\{\alpha_{t}\right\}_{t \in \mathbb{R}}$ be a flow on $\mathcal{M}$, i.e. a $\sigma$-weakly continuous one parameter group of $*$-automorphisms of $\mathcal{M}$. For each element $X \in \mathcal{M}$ and a function $f \in L^{1}(\mathbb{R})$, we define the convolution $f \underset{\alpha}{*} X$ by

$$
f *{ }_{\alpha} X=\int_{-\infty}^{+\infty} f(t) \alpha_{t}(X) d t .
$$

For $f \in L^{1}(\mathbb{R})$, let $Z(f)=\{t \in \mathbb{R}: \hat{f}(t)=0\}$, where $\hat{f}(t)=\int_{-\infty}^{+\infty} e^{-i s t} f(s) d s$ is the Fourier transform of $f$. For $X \in \mathcal{M}$, we define the Arveson spectrum of $X$ with respect to the flow $\alpha$ to be the set

$$
\bigcap\{Z(f): f \underset{\alpha}{*} X=0\}
$$

and denote it by $s p_{\alpha}(X)$. For any subset $S$ of $\mathbb{R}$ we define the spectral subspace $M^{\alpha}(S)$ to be the $\sigma$-weak closure of the set $\left\{X \in \mathcal{M}: s p_{\alpha}(X) \subset S\right\}$. We refer the readers to [3, 4, 13, 14, for the elementary properties of spectra and spectral subspaces. Put $H^{\infty}(\alpha)=M^{\alpha}([0, \infty))$ and $H_{0}^{\infty}(\alpha)=M^{\alpha}((0, \infty))$. It is known that $H_{0}^{\infty}(\alpha)$ is a two-sided ideal of $H^{\infty}(\alpha)$. Let $\mathfrak{D}=H^{\infty}(\alpha) \cap\left(H^{\infty}(\alpha)\right)^{*}$ be the fixed point subalgebra of $\alpha$. We recall that $\mathcal{M}$ is said to be $\mathbb{R}$-finite relative to $\alpha$ if there is a separating family of $\alpha$-invariant normal states on $\mathcal{M}$. At the opposite extreme, we say that $\mathcal{M}$ is completely non- $\mathbb{R}$-finite relative to $\alpha$ in case there are no invariant normal states.

Lemma 4. If $\mathcal{M}$ is completely non- $\mathbb{R}$-finite relative to $\alpha$, then $H^{\infty}(\alpha)=H_{0}^{\infty}(\alpha)$. 
Proof. Since $\mathcal{M}$ is $\sigma$-finite, without loss of generality by choosing an appropriate representation for $\mathcal{M}$, we may assume that $\mathcal{M}$ has a cyclic and separating vector in $\mathcal{H}$.

If $H^{\infty}(\alpha) \neq H_{0}^{\infty}(\alpha)$, then there is an element $f \in \mathcal{M}_{*}$ such that $f(A)=0$ for all $A \in H_{0}^{\infty}(\alpha)$ and $f(T) \neq 0$ for some $T \in H^{\infty}(\alpha)$. Since $\mathcal{M}$ has a separating vector in $\mathcal{H}$, there are vectors $x, y \in \mathcal{H}$ such that $f(A)=(A x, y)$ for all $A \in \mathcal{M}$ by Proposition 7.4.5 and Corollary 7.3.3 in [12. Let $\mathfrak{M}=\left[A x: A \in H^{\infty}(\alpha)\right]$ (resp. $\left.\mathfrak{M}_{0}=\left[A x: A \in H_{0}^{\infty}(\alpha)\right]\right)$ be the closed subspace generated by $\left\{A x: A \in H^{\infty}(\alpha)\right\}$ (resp. $\left\{A x: A \in H_{0}^{\infty}(\alpha)\right\}$ ) of $\mathcal{H}$. Then both $\mathfrak{M}$ and $\mathfrak{M}_{0}$ are invariant subspaces for $H^{\infty}(\alpha)$. We have $\mathfrak{M}_{0} \varsubsetneqq \mathfrak{M}$ since $(T x, y) \neq 0$ for some $T \in H^{\infty}(\alpha)$ and $(T x, y)=0$ for all $T \in H_{0}^{\infty}(\alpha)$. It is trivial that $H_{0}^{\infty}(\alpha) \mathfrak{M} \subseteq \mathfrak{M}_{0}$ since $H_{0}^{\infty}(\alpha)$ is a two-sided ideal of $H^{\infty}(\alpha)$. On the other hand, since $\mathcal{M}$ is completely non- $\mathbb{R}$-finite relative to $\alpha$, by Corollary 5.7 in [14, $\mathfrak{M}$ is completely normalized in the sense of Definition 5.1 in [14, that is,

$$
\mathfrak{M}=\bigwedge_{s<0}\left[M^{\alpha}([s, \infty)) \mathfrak{M}\right]=\bigvee_{s>0}\left[M^{\alpha}([s, \infty)) \mathfrak{M}\right] .
$$

Note that $M^{\alpha}([s, \infty)) \subseteq H_{0}^{\infty}(\alpha)$ for all $s>0$. We then have that $\left[M^{\alpha}([s, \infty)) \mathfrak{M}\right] \subseteq$ $\mathfrak{M}_{0}$ for all $s>0$ which implies that $\mathfrak{M} \subseteq \mathfrak{M}_{0}$. This is a contradiction. Hence $H^{\infty}(\alpha)=H_{0}^{\infty}(\alpha)$. The proof is complete.

We recall that the crossed product $\mathcal{M} \rtimes_{\alpha} \mathbb{R}$ determined by $\mathcal{M}$ and $\alpha$ is the von Neumann algebra on the Hilbert space $L^{2}(\mathbb{R}, \mathcal{H})$ generated by the operators $\pi(X)$, $X \in \mathcal{M}$, and $\lambda(s), s \in \mathbb{R}$, defined by the equations

$$
(\pi(X) f)(t)=\alpha_{-t}(X) f(t), \quad f \in L^{2}(\mathbb{R}, \mathcal{H}), t \in \mathbb{R},
$$

and

$$
(\lambda(s) f)(t)=f(t-s), \quad f \in L^{2}(\mathbb{R}, \mathcal{H}), t \in \mathbb{R} .
$$

It is clear that $\pi\left(\alpha_{t}(X)\right)=\lambda(t) \pi(X) \lambda(t)^{*}$ for all $X \in \mathcal{M}$ and $t \in \mathbb{R}$. For any $Y \in \mathcal{M} \rtimes_{\alpha} \mathbb{R}$, we define $\beta_{t}(Y)=\lambda(t) Y \lambda(t)^{*}, \forall t \in \mathbb{R}$. Then $\beta=\left\{\beta_{t}\right\}_{t \in \mathbb{R}}$ is an inner flow on $\mathcal{M} \rtimes_{\alpha} \mathbb{R}$. We know that $H^{\infty}(\beta)$ is a nest subalgebra in $\mathcal{M} \rtimes_{\alpha} \mathbb{R}$ by Theorem 4.2.3 in [14.

Let $\mathcal{A}$ be the $\sigma$-weakly closed subalgebra of $\mathcal{M} \rtimes_{\alpha} \mathbb{R}$ generated by $\{\pi(X): X \in$ $\left.H^{\infty}(\alpha)\right\}$ and $\{\lambda(t): t \in \mathbb{R}\}$. Since $\pi\left(\alpha_{t}(X)\right)=\beta_{t}(\pi(X))$ for $X \in \mathcal{M}$ and $t \in \mathbb{R}, \mathcal{A}$ is a subalgebra of $H^{\infty}(\beta)$. It is noted that $\mathcal{A}+\mathcal{A}^{*}$ is $\sigma$-weakly dense in $\mathcal{M} \rtimes_{\alpha} \mathbb{R}$ since $H^{\infty}(\alpha)+H^{\infty}(\alpha)^{*}$ is $\sigma$-weakly dense in $\mathcal{M}$ by Theorem 3.15 in 14 .

Lemma 5. $H_{0}^{\infty}(\beta) \subset \mathcal{A}$.

Proof. We know that $H_{0}^{\infty}(\beta)$ is the $\sigma$-weak closure of the set $\left\{X \in \mathcal{M} \rtimes_{\alpha} \mathbb{R}\right.$ : $s p_{\beta}(X)$ is compact in $\left.(0,+\infty)\right\}$ by Lemma 2.8 in [16. Let $X \in \mathcal{M} \rtimes_{\alpha} \mathbb{R}$ be such that $s p_{\beta}(X)$ is compact in $(0,+\infty)$. Choose $f \in L^{1}(\mathbb{R})$ with compactly supported Fourier transform such that support supp $\hat{f}$ of $\hat{f}$ is in $(0, \infty)$ and such that $f * X=X$. Note that since $\mathcal{A}+\mathcal{A}^{*}$ is $\sigma$-weakly dense in $\mathcal{M} \rtimes_{\alpha} \mathbb{R}$, there are nets $\left\{A_{i}\right\},\left\{B_{i}\right\}$ in $\mathcal{A}$ such that $\lim _{i}\left(A_{i}+B_{i}^{*}\right)=X \sigma$-weakly. It follows that $\lim _{i}\left(f \underset{\beta}{*} A_{i}+f \underset{\beta}{*} B_{i}^{*}\right)=$ $f \underset{\beta}{*} X=X$. However, we have $f_{\beta}^{*} B_{i}^{*}=0$ since $s p_{\beta}\left(f \underset{\beta}{*} B_{i}^{*}\right) \subset \operatorname{supp} \hat{f} \cap s p_{\beta}\left(B_{i}^{*}\right)=\emptyset$. Note that $\mathcal{A}$ is $\left\{\beta_{t}\right\}_{t \in \mathbb{R}}$ invariant, it follows that $f_{\beta}^{*} A_{i} \in \mathcal{A}$ and then $X \in \mathcal{A}$. The proof is complete. 
The next result might be known, but we were unable to find a reference for it.

Lemma 6. If $\mathcal{M}$ is $\mathbb{R}$-finite relative to $\alpha$, then $\mathcal{M} \rtimes_{\alpha} \mathbb{R}$ is $\mathbb{R}$-finite relative to $\beta$.

Proof. By considering a covariant representation of the pair $(\mathcal{M}, \alpha)$ in the sense of Definition 2.5 and Proposition 2.6 in [14, we may assume that $\alpha=\left\{\alpha_{t}\right\}_{t \in \mathbb{R}}$ is implemented by a continuous unitary representation $t \rightarrow U_{t}$ of $\mathbb{R}$ on $\mathcal{H}$, that is, $\alpha_{t}(X)=U_{t} X U_{t}^{*}$ for all $x \in \mathcal{M}$. Then by Definition 13.2.6 in 12, the crossed product $\mathcal{M} \rtimes_{\alpha} \mathbb{R}$ is a von Neumann algebra on $L^{2}(\mathcal{R}, \mathcal{H})\left(=\mathcal{H} \otimes L^{2}(\mathbb{R})\right)$ generated by $\{A \otimes I: a \in \mathcal{M}\}$ and $\left\{U_{t} \otimes l_{t}: t \in \mathbb{R}\right\}$, where $l_{t}$ is the shift operator on $L^{2}(\mathbb{R})$ defined by $\left(l_{t} f\right)(s)=f(s-t), t, s \in \mathbb{R}, f \in L^{2}(\mathbb{R})$. It is known that $\beta$ is implemented by $\left\{U_{t} \otimes l_{t}\right\}_{t \in \mathbb{R}}$.

Since $L^{2}(\mathbb{R})$ is separable, there is an orthonormal basis $\left\{e_{n}: n=1,2, \cdots\right\}$ of $L^{2}(\mathbb{R})$. If we define $\mu(T)=\sum_{n=1}^{\infty} \frac{1}{2^{n}}\left(T e_{n}, e_{n}\right), \forall T \in \mathcal{B}\left(L^{2}(\mathbb{R})\right)$, then $\mu$ is a faithful normal state of $\mathcal{B}\left(L^{2}(\mathbb{R})\right)$. Let $\varphi$ be a faithful normal state of $\mathcal{M}$ such that $\varphi \circ \alpha_{t}=\varphi$ for all $t \in \mathbb{R}$. It follows from Proposition 11.2.7 in 12 that $\varphi \bar{\otimes} \mu$ is a normal state on $\mathcal{M} \bar{\otimes} \mathcal{B}\left(L^{2}(\mathbb{R})\right) \supset \mathcal{M} \rtimes_{\alpha} \mathbb{R}$. We claim that $\varphi \bar{\otimes} \mu$ is faithful. In fact, we way identify $\mathcal{M} \bar{\otimes} \mathcal{B}\left(L^{2}(\mathbb{R})\right)$ with $\left\{\left(A_{i j}\right) \in \mathcal{B}\left(\mathcal{H} \otimes L^{2}(\mathbb{R})\right): A_{i j} \in \mathcal{M}\right\}$ by Remark 11.2.3 in [12. For any $\left(A_{i j}\right) \in \mathcal{M} \bar{\otimes} \mathcal{B}\left(L^{2}(\mathbb{R})\right)$, we define $\psi\left(\left(A_{i j}\right)\right)=\sum_{n=1}^{\infty} \frac{1}{2^{n}} \varphi\left(A_{n n}\right)$. Then $\psi$ is a faithful normal state on $\mathcal{M} \bar{\otimes} \mathcal{B}\left(L^{2}(\mathbb{R})\right)$. Since for any $A \in \mathcal{M}$ and $T \in$ $\mathcal{B}\left(L^{2}(\mathbb{R})\right), A \otimes T$ identifies with $\left(t_{i j} A\right)$, where $t_{i j}=\left(T e_{j}, e_{i}\right)$ for all $i, j=1,2, \cdots$, $\varphi \bar{\otimes} \mu(A \otimes T)=\varphi(A) \mu(T)=\psi\left(\left(t_{i j} A\right)\right)$. It follows that $\varphi \bar{\otimes} \mu=\psi$ and then $\varphi \bar{\otimes} \mu$ is faithful.

We note that if $A \in \mathcal{M}$, then $\varphi \bar{\otimes} \mu\left(\beta_{s}(A \otimes I)\right)=\varphi \bar{\otimes} \mu(A \otimes I)$ for all $s \in \mathbb{R}$. On the other hand, $\beta_{t}\left(U_{s} \otimes l_{s}\right)=U_{s} \otimes l_{s}$; then $\varphi \bar{\otimes} \mu\left(\beta_{t}\left(U_{s} \otimes l_{s}\right)\right)=\varphi \bar{\otimes} \mu\left(U_{s} \otimes l_{s}\right)$, $s, t \in \mathbb{R}$. We now have that $\varphi \bar{\otimes} \mu$ is a faithful normal state on $\mathcal{M} \rtimes_{\alpha} \mathbb{R}$ such that $\varphi \bar{\otimes} \mu \circ \beta_{s}=\varphi \bar{\otimes} \mu$ for all $s \in \mathbb{R}$. It follows that $\mathcal{M} \rtimes_{\alpha} \mathbb{R}$ is $\mathbb{R}$-finite relative to $\beta$. The proof is complete.

Lemma 7. $H^{\infty}(\beta)=\mathcal{A}$.

Proof. We first assume that $\mathcal{M}$ is $\mathbb{R}$-finite relative to $\left\{\alpha_{t}\right\}_{t \in \mathbb{R}}$. Then there is a faithful normal expectation $\Phi$ from $\mathcal{M}$ onto $\mathcal{D}$. Now there is a faithful normal expectation $\Psi$ from $\mathcal{M} \rtimes_{\alpha} \mathbb{R}$ onto $\mathcal{N}$ such that $\Psi(\pi(A))=\pi(\Phi(A))$ for all $A \in \mathcal{M}$ by Lemma 6 , where $\mathcal{N}$ is the fixed point algebra of $\beta$. We have that $\mathcal{N}$ is generated by $\{\pi(A): A \in \mathfrak{D}\}$ and $\{\lambda(t): t \in \mathbb{R}\}$. In fact, take any $D \in \mathcal{N}$. There is a net $A_{i}=\sum_{j=1}^{n_{i}} \pi\left(X_{j}^{i}\right) \lambda\left(t_{j}^{i}\right)$ such that $\lim _{i} A_{i}=D \sigma$-weakly, where $X_{j}^{i} \in \mathcal{M}$ and $t_{j}^{i} \in \mathbb{R}$. We then have

$$
\begin{aligned}
D & =\Psi(D)=\lim _{i} \Psi\left(A_{i}\right)=\lim _{i} \sum_{j=1}^{n_{i}} \Psi\left(\pi\left(X_{j}^{i}\right)\right) \lambda\left(t_{j}^{i}\right) \\
& =\lim _{i} \sum_{j=1}^{n_{i}} \pi\left(\Phi\left(X_{j}^{i}\right)\right) \lambda\left(t_{j}^{i}\right) .
\end{aligned}
$$

It now follows that $\mathcal{N}=H^{\infty}(\beta) \cap H^{\infty}(\beta)^{*}=\mathcal{A} \cap \mathcal{A}^{*}$. We know that $H_{0}^{\infty}(\beta) \subset \mathcal{A}$ by Lemma 5. Thus $H^{\infty}(\beta)=\mathcal{N}+H_{0}^{\infty}(\beta) \subset \mathcal{A}$ and therefore $H^{\infty}(\beta)=\mathcal{A}$.

For the general case, there is a projection $E$ in the center of $\mathfrak{D}$ such that $E \mathcal{M} E$ is $\mathbb{R}$-finite, while $(I-E) \mathcal{M}(I-E)$ is completely non- $\mathbb{R}$-finite relative to $\alpha$ from Remark 
3.4 in [14. We note that in this case $\pi(E)\left(\mathcal{M} \rtimes_{\alpha} \mathbb{R}\right) \pi(E)$ is $\mathbb{R}$-finite by Lemma 6 , and $\left(I-\pi(E)\left(\mathcal{M} \rtimes_{\alpha} \mathbb{R}\right)(I-\pi(E))\right.$ is completely non- $\mathbb{R}$-finite relative to $\beta$. By considering $\alpha$ restricted on $E \mathcal{M} E$, we have $\pi(E) H^{\infty}(\beta) \pi(E) \subset \pi(E) \mathcal{A} \pi(E) \subset \mathcal{A}$ by Lemma 6 again. On the other hand, if we consider $\alpha$ on $(I-E) \mathcal{M}(I-E)$, then we have $(I-\pi(E)) H^{\infty}(\beta)(I-\pi(E))=(I-\pi(E)) H_{0}^{\infty}(\beta)(I-\pi(E)) \subset H_{0}^{\infty}(\beta)$. In particular, $I-\pi(E) \in H_{0}^{\infty}(\beta)$ and then $(I-\pi(E)) H^{\infty}(\beta)+H^{\infty}(\beta)(I-\pi(E)) \subset H_{0}^{\infty}(\beta) \subset \mathcal{A}$. Thus we have $H^{\infty}(\beta) \subset \mathcal{A}$, and the proof is complete.

Theorem 2. The commutant of $H^{\infty}(\alpha)$ is self-adjoint, that is, $\left(H^{\infty}(\alpha)\right)^{\prime}=\mathcal{M}^{\prime}$.

Proof. Let $\beta=\left\{\beta_{t}\right\}_{t \in \mathbb{R}}$ be as above. We recall that $H^{\infty}(\beta)$ is a nest subalgebra of $\mathcal{M} \rtimes_{\alpha} \mathbb{R}$. Then the commutant of $H^{\infty}(\beta)$ is self-adjoint by Theorem 2.5 in [6. Let $X \in\left(H^{\infty}(\alpha)\right)^{\prime}$. Define an operator $\hat{X}$ on $L^{2}(\mathbb{R}, \mathcal{H})$ by

$$
(\hat{X} \xi)(t)=X \xi(t), \quad \forall \xi \in L^{2}(\mathbb{R}, \mathcal{H}) .
$$

Then it is trivial that $\hat{X}$ is bounded. We claim that $\hat{X} \in\left(H^{\infty}(\beta)\right)^{\prime}$. By Lemma 7 , it is sufficient to show that $\hat{X} \in \mathcal{A}^{\prime}$. For any $Y \in H^{\infty}(\alpha)$ we have

$$
\begin{aligned}
& (\hat{X} \pi(Y) \xi)(t)=X \alpha_{-t}(Y) \xi(t)=\alpha_{-t}(Y) X \xi(t) \\
& =\alpha_{-t}(Y)(\hat{X} \xi)(t)=(\pi(Y) \hat{X} \xi)(t) .
\end{aligned}
$$

Then $\hat{X} \pi(Y)=\pi(Y) \hat{X}$. On the other hand, for any $s \in \mathbb{R}$,

$$
\begin{aligned}
& (\hat{X} \lambda(s) \xi)(t)=(\hat{X} \xi)(t-s) \\
& =\lambda(s)(\hat{X} \xi)(t)=(\lambda(s) \hat{X})(t) .
\end{aligned}
$$

It follows that $\lambda(s) \hat{X}=\hat{X} \lambda(s)$ for any $s \in \mathbb{R}$. We thus have $\hat{X} \in\left(H^{\infty}(\beta)\right)^{\prime}$. By Theorem 2.5 in [6], $(\hat{X})^{*} \in\left(H^{\infty}(\beta)\right)^{\prime}$. In particular, $(\hat{X})^{*}$ commutes with $\pi(Y)$ for any $Y \in H^{\infty}(\alpha)$. Note that $(\hat{X})^{*}=\left(X^{*}\right)^{\text {. }}$. Given $u$ in $\mathcal{H}$, let $f$ be a continuous function in $L^{2}(\mathbb{R})$ such that $f(0)=1$ and let $\xi(t)=f(t) u$. Then $\xi \in L^{2}(\mathbb{R}, \mathcal{H})$ and

$$
\begin{aligned}
& \left(\left(X^{*}\right) \pi(Y) \xi\right)(t)=X^{*} \alpha_{-t}(Y) \xi(t)=X^{*} \alpha_{-t}(Y) f(t) u \\
& =\left(\pi(Y)\left(X^{*}\right) \xi\right)(t)=\alpha_{-t}(Y) X^{*} f(t) u .
\end{aligned}
$$

When $t=0$, we have $X^{*} Y u=Y X^{*} u$ for all $u \in \mathcal{H}$. Hence $X^{*} \in\left(H^{\infty}(\alpha)\right)^{\prime}$ and $\left(H^{\infty}(\alpha)\right)^{\prime}=\mathcal{M}^{\prime}$. The proof is complete.

Remark 1 . We know that if $\mathcal{A}$ is either a subdiagonal algebra or an analytic operator algebra of $\mathcal{M}$, then we have that $\mathcal{A}+\mathcal{A}^{*}$ is $\sigma$-weakly dense in $\mathcal{M}$. However, if we assume that a subalgebra only satisfies this condition, it may not follow that the algebraic commutant is self-adjoint. For example, from Corollary 1.4 in [1, we know that there is a subalgebra $\mathcal{A}$ of $\mathcal{B}(\mathcal{H})$ such that $\mathcal{A}+\mathcal{A}^{*}$ is $\sigma$-weakly dense in $\mathcal{B}(\mathcal{H})$ and such that $\mathcal{A}$ is similar to a proper von Neumann subalgebra of $\mathcal{B}(\mathcal{H})$. It easily follows that the algebraic commutant of $\mathcal{A}$ is not self-adjoint.

Remark 2. We considered two classes of non-self-adjoint operator algebras, subdiagonal algebras and analytic operator algebras determined by flows in von Neumann algebras. If $\mathcal{H}$ is finite dimensional, we know that these two classes of operator algebras are nest subalgebras of von Neumann algebras (cf. Theorem 2.1 in [11]). However, if $\mathcal{H}$ is infinite dimensional, these two classes are different. The analytic operator algebra $H^{\infty}(\alpha)$ determined by a flow $\alpha$ is a maximal subdiagonal algebra if and only if the flow $\alpha$ is $\mathbb{R}$-finite. There are subdiagonal algebras which are not 
analytic operator algebras determined by any flows. We refer the readers to see some examples given in 8 .

\section{ACKNOWLEDGEMENT}

The authors thank the referee for the valuable comments and suggestions and for pointing out reference [15] which was useful for the revision of this paper.

\section{REFERENCES}

[1] M. Anoussis, A. Katavolos and M. Lambrou, On the reflexive algebra with two invariant subspaces, J. Operator Theory, 130(1993), 267-299. MR1305508(95i:47082)

[2] W. B. Arveson, Analyticity in operator algebras, Amer. J. Math., 89(1967), 578-642. MR0223899 (36:6946)

[3] W. B. Arveson, Operator algebras and measure preserving automorphisms, Acta. Math., 118(1967), 95-109. MR0210866 (35:1751)

[4] W. B. Arveson, On groups of automorphisms of operator algebras, J. Funct. Anal., 15(1974), 217-243. MR.0348518 (50:1016)

[5] R. Exel, Maximal subdiagonal algebras, Amer. J. Math., 110(1988), 775-782. MR0955297 (90b:46114)

[6] F. Gilfeather and D. R. Larson, Nest-subalgebras of von Neumann algebras: Commutants modulo compacts and distance estimates, J. Operator Theory, 7(1982), 279-302. MR0658614 (84g:47040)

[7] U. Haagerup, Non-commutative integration theory, unpublished manuscript, 1980.

[8] G. X. Ji, Relative lattices of certain analytic operator algebras, Houston J. Math., 28(2002), 183-191. MR.1876948 (2002j:47123)

[9] G. X. Ji and H. K. Du, Subdiagonal algebras with the factorization property, Acta Math. Sinica, 46(2003), 883-890. MR2025391 (2004j:46080)

[10] G. X. Ji, T. Ohwada and K.-S. Saito, Certain structure of subdiagonal algebras, J. Operator Theory, 39 (1998), 309-317. MR.1620570 (99b:46093)

[11] G. X. Ji, T. Ohwada and K.-S. Saito, Triangular forms of subdiagonal algebras, Hokkaido Math. J., 27 (1998), 545-552. MR.1662955 (99m:47053)

[12] R. V. Kadison and J. R. Ringorse, Fundamentals of the theory of operator algebras, Vol. II, Academic Press, Orlando, 1986. MR0859186 (88d:46106)

[13] S. Kawamura and J. Tomiyama, On subdiagonal algebras associated with flows in operator algebras, J. Math. Soc. Japan, 29(1977), 73-90. MR0454650 (56:12899)

[14] R. Loebl and P. S. Muhly, Analyticity and flows in von Neumann algebras, J. Funct. Anal., 29(1978), 214-252. MR0504460 (81h:46080)

[15] A.I. Loginov and V.S. Shulman, Hereditary and Intermediate Reflexivity of $w^{*}$-algebras, Izv. Akad. Nauk SSSR, Ser. Mat. Tom 39 (1975), No. 6: Mat. USSR Izvestija, 9(1975), 1189-1201. MR.0405124(538919)

[16] B. Solel, Maximality of analytic operator algebras, Israel J. Math., 62(1988), 63-89. MR0947830 (89i:46071)

[17] Q. Xu, On the maximality of subdiagonal algebras, J. Operator Theory, 54 (2005), 137-146. MR2168864

College of Mathematics and Information Science, Shaanxi Normal University, Xian, 710062, People's Republic of China

E-mail address: gxji@snnu.edu.cn

Department of General Science, Tsuruoka National College of Technology, TSURUOKA, 997-8511, JAPAN

E-mail address: ohwada@tsuruoka-nct.ac.jp

Department of Mathematics, Faculty of Science, Nitgata University, Niigata, 9502181, JAPAN

E-mail address: saito@math.sc.niigata-u.ac.jp 